\title{
Regulatory RNA at the crossroads of carbon and nitrogen metabolism in photosynthetic cyanobacteria
}

\author{
Alicia M. Muro-Pastor ${ }^{\mathrm{a}}$, Wolfgang R. Hess ${ }^{\mathrm{b}, \mathrm{c}, *}$ \\ a Instituto de Bioquímica Vegetal y Fotosíntesis, Consejo Superior de Investigaciones Científicas and Universidad de Sevilla, Américo Vespucio 49, E-41092 Sevilla, Spain \\ ${ }^{\mathrm{b}}$ University of Freiburg, Faculty of Biology, Genetics and Experimental Bioinformatics, Schänzlestr. 1, D-79104 Freiburg, Germany \\ ${ }^{\mathrm{c}}$ University of Freiburg, Freiburg Institute for Advanced Studies, Albertstr. 19, D-79104 Freiburg, Germany
}

\section{A R T I C L E I N F O}

\section{Keywords:}

Antisense RNAs

Cyanobacteria

Heterocysts

Post-transcriptional regulation

Riboswitches

sRNAs

\begin{abstract}
A B S T R A C T
Cyanobacteria are photosynthetic bacteria that populate widely different habitats. Accordingly, cyanobacteria exhibit a wide spectrum of lifestyles, physiologies, and morphologies and possess genome sizes and gene numbers which may vary by up to a factor of ten within the phylum. Consequently, large differences exist between individual species in the size and complexity of their regulatory networks. Several non-coding RNAs have been identified that play crucial roles in the acclimation responses of cyanobacteria to changes in the environment. Some of these regulatory RNAs are conserved throughout the cyanobacterial phylum, while others exist only in a few taxa. Here we give an overview on characterized regulatory RNAs in cyanobacteria, with a focus on regulators of photosynthesis, carbon and nitrogen metabolism. However, chances are high that these regulators represent just the tip of the iceberg.
\end{abstract}

\section{Introduction}

\subsection{Cyanobacteria in their natural environment}

Cyanobacteria are the only bacteria that perform oxygenic photosynthesis. Therefore, they are of paramount ecological importance as primary producers at the bottom of the global food chain. This is illustrated by estimations that a single cyanobacterial genus, Prochlorococcus, is with a population size of $\sim 10^{27}$ cells the most abundant photosynthetic organism on Earth $[1,2]$. In addition to the capability of cyanobacteria for the fixation of inorganic carbon $\left(\mathrm{C}_{\mathrm{i}}\right)$ through the photosynthetic Calvin-Benson-Bassham (CBB) cycle [3], diazotrophic species contribute substantial amounts of combined nitrogen into the biosphere by converting $\mathrm{N}_{2}$ into ammonia, a process which is known as nitrogen fixation [4].

Cyanobacteria are not restricted to aquatic environments but occupy diverse ecological niches. While many thrive in marine and freshwater, others belong to terrestrial ecosystems and some even live in extreme environments like deserts, the polar regions or thermal waters. Cyanobacteria are not always free-living but many are essential for structuring complex microbial communities, such as in stromatolites [5], coastal microbial mats [6] and desert biological soil crusts [7]. There are endolithic forms [8] and others are symbionts of certain higher plants [9], the water-fern Azolla [10], fungi (to form lichens) or several different unicellular eukaryotes [11-14].

Because of the vastly different lifestyles, cyanobacteria vary substantially with regard to morphologies, physiology, genome size and ultimately, the machinery for the control of gene expression. For example, the number of protein-coding genes varies between ca. 1200 for Candidatus Atelocyanobacterium thalassa (UCYN-A), a unicellular marine $\mathrm{N}_{2}$-fixing symbiont [15] and more than 12,300 for the multicellular $\mathrm{N}_{2}$-fixing Scytonema hofmanni PCC 7110 [16]. Cyanobacterial metabolism is postulated to possess substantial plasticity in responding to environmental cues. Most of these cues are common also to other microbes, but the predominance of oxygenic photosynthesis-driven primary metabolism presents an additional set of regulatory challenges, mostly related to light and iron availability, setting cyanobacteria apart from other bacteria. Indeed, the machinery for oxygenic photosynthesis is one of the most iron-rich cellular systems. The full complement of photosystem I (PSI) complexes of a unicellular cyanobacterium were estimated to contain about one order of magnitude more iron than an average $E$. coli cell $[17,18]$. Consequently, oxygenic photosynthesis is fundamentally vulnerable to phases of iron starvation, a situation that occurs frequently in nature [19]. In addition to light and iron, limitations in the availability of the macronutrients $\mathrm{C}_{\mathrm{i}}$ and $\mathrm{N}$ are major challenges for cyanobacteria.

To summarize, while distinct cyanobacterial lineages may differ in many aspects from one another, they are monophyletic as a phylum and

\footnotetext{
* Corresponding author at: University of Freiburg, Faculty of Biology, Genetics and Experimental Bioinformatics, Schänzlestr. 1, D-79104 Freiburg, Germany

E-mail address: wolfgang.hess@biologie.uni-freiburg.de (W.R. Hess).
} 
face certain challenges that apply to them all alike. Therefore, there are large differences in the complexity and composition of the regulatory networks between individual species but also some components that are shared by many different cyanobacteria and set them apart from other groups of bacteria.

\subsection{The control of carbon and nitrogen assimilation in cyanobacteria:} basics

For the growth of cyanobacteria, $\mathrm{C}_{\mathrm{i}}$ and $\mathrm{N}$ are macronutrients of central importance. Intriguingly, the concentrations of available $\mathrm{C}_{\mathrm{i}}$ and $\mathrm{N}$ molecules influence the capacity for assimilation of the other macronutrient. There is only a limited capacity to store an excess of fixed carbon if nitrogen is a limiting factor and, similarly, a limited supply of carbon skeletons will limit the assimilation of nitrogen. This relationship is known as the $\mathrm{C} / \mathrm{N}$ balance. It is for this reason that the cell enhances carbon uptake if nitrogen becomes easily available and, vice versa, why carbon limitation lowers the uptake and assimilation of nitrogen. The genetic responses linked to these aspects, with a focus on filamentous, heterocystous cyanobacteria such as Anabaena, have recently been reviewed [20].

One point where carbon and nitrogen assimilation pathways intersect is the C5 compound 2-oxo-glutarate (2-OG). Whereas 2-OG is a central intermediate of the tricarboxylic acid (TCA) cycle, it is also a substrate of the glutamate synthase (glutamine oxoglutarate aminotransferase, GOGAT), which catalyzes the transfer of the amino group from glutamine to 2-OG, yielding two molecules of glutamate. This glutamate then is the substrate for the key enzyme of nitrogen assimilation in cyanobacteria, glutamine synthetase (GS, encoded by the gene $\operatorname{gln} A$ ), which catalyzes the synthesis of glutamine from glutamate and ammonium. Depending on the nitrogen and carbon availability, the level of 2-OG varies, making this metabolite an excellent indicator of nitrogen status and the $\mathrm{C} / \mathrm{N}$ ratio $[21,22]$. Indeed, the levels of 2-OG are sensed by several proteins involved in the regulation of nitrogen assimilation. The activity of the main transcriptional regulator of nitrogen assimilation, NtcA, is modulated by binding 2-OG directly [23,24]. Under nitrogen-replete conditions (e.g., in the presence of $\mathrm{NH}_{4}{ }^{+}$), the 2-OG level is low and NtcA is present in a less active form, with low affinity to its target promoters [25]. Therefore, it is physiologically meaningful to stimulate NtcA activity when the 2-OG level is increasing due to a lack of available nitrogen. However, 2-OG is also sensed by two other regulators of nitrogen assimilation in cyanobacteria, the proteins PipX and $\mathrm{P}_{\mathrm{II}}$. According to the nitrogen status, PipX can interact with either NtcA or $\mathrm{P}_{\mathrm{II}}$, depending on the 2-OG level [26,27]. In the complex with NtcA it enhances the binding affinity of this complex to target promoters further [28]. $\mathrm{P}_{\text {II }}$ proteins, more generally, regulate nitrogen assimilation reactions by sensing the metabolic state of the cells through binding of 2-OG and ATP/ADP as effector molecules [29]. Extending these findings, SbtB, another member of the PII superfamily in cyanobacteria, has recently been shown to function as a sensor for the levels of intracellular inorganic carbon $\left(\mathrm{C}_{\mathrm{i}}\right)$ via cAMP binding [30]. The metabolite 2-OG was reported as effector for another regulatory protein, where it acts together with $\mathrm{NADP}^{+}$as co-repressor of the transcriptional regulator of carbon uptake and assimilation, NdhR/ CcmR [31]. Other metabolites which exert regulatory functions in the context of $\mathrm{C} / \mathrm{N}$ metabolism in cyanobacteria are 2-phosphoglycolate (2PG) and ribulose 1,5-bisphosphate, which stimulate promoter-binding of the activator CmpR [32]. Furthermore, upon binding to NdhR, 2-PG functions as inducer of carbon uptake and assimilation, directly counteracting the function of 2-OG [33]. Therefore, the key intermediates 2OG from the TCA cycle and 2-PG from photorespiration connect cyanobacterial carbon and nitrogen metabolism at a central point (recently reviewed by Zhang et al. [34]). In addition to ATP/ADP, NADP ${ }^{+}$and the non-nitrogenous metabolites 2-OG, 2-PG and ribulose 1,5-bisphosphate, glutamine has recently been recognized as an important metabolite in signaling the $\mathrm{C} / \mathrm{N}$ status, sensed through a unique riboswitch
[35].

Recent findings have shown that several different regulatory RNAs are involved in the control of carbon and nitrogen assimilation in cyanobacteria. In the following sections of this review we are summarizing recent findings on the involvement of regulatory RNAs in the control of iron, carbon and nitrogen assimilation in cyanobacteria as well as on the utilization of light, and place these findings in their respective physiological and developmental context.

\subsection{Transcription of non-coding RNAs is abundant in cyanobacteria}

Several hundreds of potentially regulatory non-coding (nc)RNAs such as trans-acting small (sRNAs), antisense RNAs (asRNAs) overlapping other transcripts in cis as well as riboswitches have been identified in cyanobacteria. While the first individual candidates for regulatory RNAs were identified by computational prediction and experimental validation [36-38], transcriptomic approaches have been yielding a much more comprehensive picture.

In the unicellular model strains Synechocystis sp. PCC 6803 (from here Synechocystis 6803) and Synechocystis sp. PCC 6714 (from here Synechocystis 6714), 371 and 306 transcriptional start sites (TSS) were assigned to the transcription of sRNAs, which corresponded to $5.5 \%$ and $6 \%$ of all TSS, respectively [39-41]. Similar numbers were reported for the fast-growing cyanobacterial model strain, Synechococcus elongatus UTEX 2973, with 1380 TSS for asRNAs and 229 nTSS from which sRNAs originate, corresponding to $4.8 \%$ of all TSS [42]. High numbers of putative sRNAs were also reported for the filamentous multicelluar, $\mathrm{N}_{2}$-fixing cyanobacteria Nostoc, Nodularia and Trichodesmium (Fig. 1). For Nostoc (Anabaena) sp. PCC 7120 (from here Nostoc 7120), 1414 nTSS were identified that give rise to likely sRNAs, corresponding to $10.3 \%$ of all TSS [43], while for Nodularia spumigena CCY9414 621 nTSS were reported, corresponding to $12.3 \%$ of all TSS $[44,45]$. The extreme was found for Trichodesmium erythraeum sp. IMS101, with 1621 nTSS that were assigned to sRNAs out of a total of 6080 TSS corresponding to $26.7 \%$ [46], which has been linked to the high share of non-coding DNA in the genome [47].

The comparison of dRNA-Seq data from eight different cyanobacteria consistently shows that only $27-51 \%$ of all TSS drive the transcription of protein-coding genes (Fig. 1). Thus, a large fraction of the transcriptional output is non-coding, even when considering differences in the study designs, in the biochemical protocols, in sequencing depths, the definition of transcript types and the fact that some sRNAs later may turn out to encode previously unrecognized small proteins [48]. Hence, there are many sRNAs, asRNAs and also some riboswitches in cyanobacteria, which likely are relevant for the regulation of photosynthetic processes, iron homeostasis and control of the $\mathrm{C} / \mathrm{N}$ balance.

\section{A set of mutually titrating pairs of sRNAs are conserved throughout the cyanobacterial phylum}

Early computational analyses identified sRNAs called Yfr1 to Yfr7 (Yfr stands for cyanobacterial functional RNA) in the marine picocyanobacterium Prochlorococcus, which later turned out to have homologs in most other cyanobacterial genomes [36,37,49-51]. The sRNAs Yfr2, Yfr3, Yfr4 and Yfr5 belong to one family of closely related sRNAs in Prochlorococcus MED4. While these four sRNAs make up the Yfr2 family in the initially studied strain, Prochlorococcus MED4, there are up to nine individual sRNAs that belong to this family in some other cyanobacteria [50]. Three copies of Yfr2 genes exist in Synechocystis 6803 and 6714 , respectively, where they were called $y f r 2 a, y f r 2 b$ and $y f r 2 c$. Although the respective sRNAs accumulate as distinct transcripts, 80, 65 and $70 \mathrm{nt}$ in length [52], only Yfr2a is transcribed from a free-standing gene, while $y f r 2 b$ and $y f r 2 c$ are fused to the coding regions of proteincoding genes constituting their $5^{\prime}$ UTRs, a genetic set-up called an 'actuaton' $[41,53]$. 


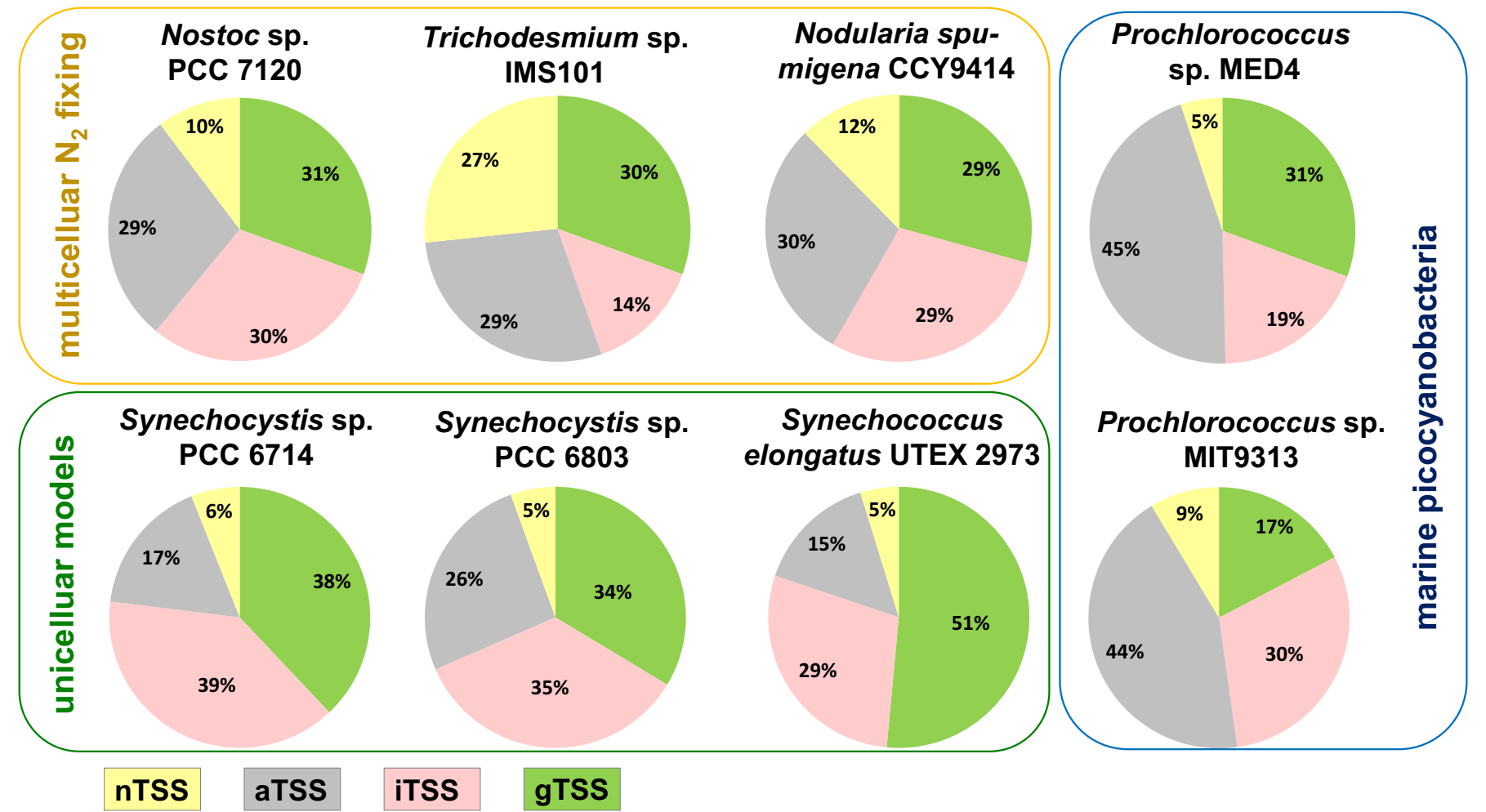

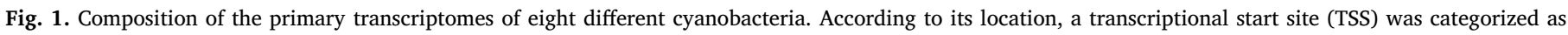

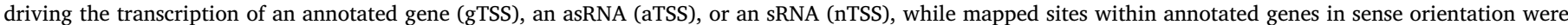

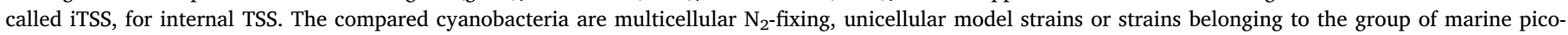

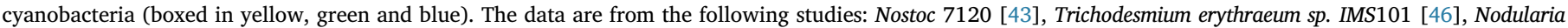

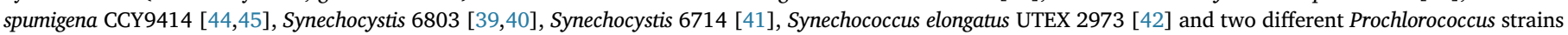
[102]. (For interpretation of the references to colour in this figure legend, the reader is referred to the web version of this article.)

A function was shown for Yfr1 in Prochlorococcus MED4 in the control of gene expression for the two major outer-membrane porins PMM1119 and PMM121 [54]. In addition, Yfr1 was suggested to control in Synechococcus elongatus PCC 6301 the expression of sbtA encoding a sodium-dependent bicarbonate transporter [55].

All Yfr1 homologs share the ultraconserved sequence motif 5'-ACUCCUCACAC-3' (Fig. 2), which in addition in several Prochlorococcus strains is present in another sRNA, called Yfr10, which hence can be considered another member of the same sRNA family [56]. The Yfr1 and Yfr2 sRNAs were suggested to interact with each other because the most conserved segments of these sRNAs have an almost perfect sequence complementarity to each other over 13 consecutive nucleotides [57]. This sequence complementarity is conserved throughout the cyanobacterial phylum and can be observed in cyanobacteria ranging from Prochlorococcus to heterocystous strains. Moreover, in predictions of secondary structures, these likely interacting sequences are located in single-stranded regions. Interaction in vitro has been shown for Yfr1 and Yfr2 from Nostoc 7120 [58].

Indeed, recent analyses in Prochlorococcus MED4 using a hybrid sRNA-target pulldown followed by sequencing (called CRAFD-Seq) showed strong enrichment for Yfr1 and Yfr10 when Yfr2 was used as bait and vice versa, in the reciprocal fishing approach, largely Yfr2 and its three homologs interacted with Yfr1 or Yfr10 [59]. In addition, a large but distinct set of mRNAs was enriched in the pull downs and are thus potential targets for sRNAs belonging to one or the other sRNA family. Of particular interest has been the finding that several mRNAs encoding proteins involved in carbon primary metabolism became enriched as potential targets of Yfr1 and Yfr10. Some of these mRNAs possess asRNAs that were found enriched as likely Yfr2 targets. One striking example is the $g l g C$ mRNA encoding glucose-1-phosphate adenylyltransferase (gene PMM0769 in Prochlorococcus MED4), which is bound by Yfr1, Yfr10 and by its asRNA, asGlgC, which in turn, was targeted by Yfr2 [59]. The collected evidence suggested that similar complex post-transcriptional regulation may exist for the gap2 gene encoding glyceraldehyde-3-phosphate dehydrogenase, pgk encoding phosphoglycerate kinase and pgmI encoding phosphoglycerate mutase, PMM1434. All these genes appear to be controlled by Yfr1/Yfr10 and by specific asRNAs, which, in turn, are titrated by Yfr2 [59]. But how would this mechanism work if sRNAs of one or the other family would just basepair, effectively neutralizing each other? A likely answer is that, while Yfr1 was constitutively expressed, two of the Yfr2 sRNA homologs became highly upregulated during the shift to HL or nitrogen starvation in Prochlorococcus MED4, the latter response controlled by the GntR family transcriptional regulator PMM1637 [60]. This has been taken as evidence that the resulting differences in the ratio between the members of each sRNA family favor regulation through either the Yfr1 or the Yfr2 arm of this mechanism (Fig. 2). Thus, the Yfr1-Yfr2 system appears to be at the core of a post-transcriptional regulatory mechanism in cyanobacteria that tunes gene expression according to the $\mathrm{C} / \mathrm{N}$ balance.

Once such a mechanism of mutually titrating sets of sRNAs, each with specific mRNA targets and involving asRNAs, had evolved, it likely became stabilized because the mutation or loss of key elements such as Yfr1 and Yfr2 sRNAs would have been detrimental (Fig. 2). Indeed, repeated attempts to delete the yfr1 gene from Synechocystis 6803 failed (W.R. Hess, unpubl. observations). On the other hand, once such a mechanism became available, additional targets might have evolved, while other target genes or target sequences might have got lost over time (Fig. 2). Hence, large variation in the gene complement influenced by the Yfr1-Yfr2 system might be expected when representatives of distant cyanobacteria are to be analyzed. In fact, a recent report shows that in Nostoc 7120, Yfr1 is an important regulator of cell wall 


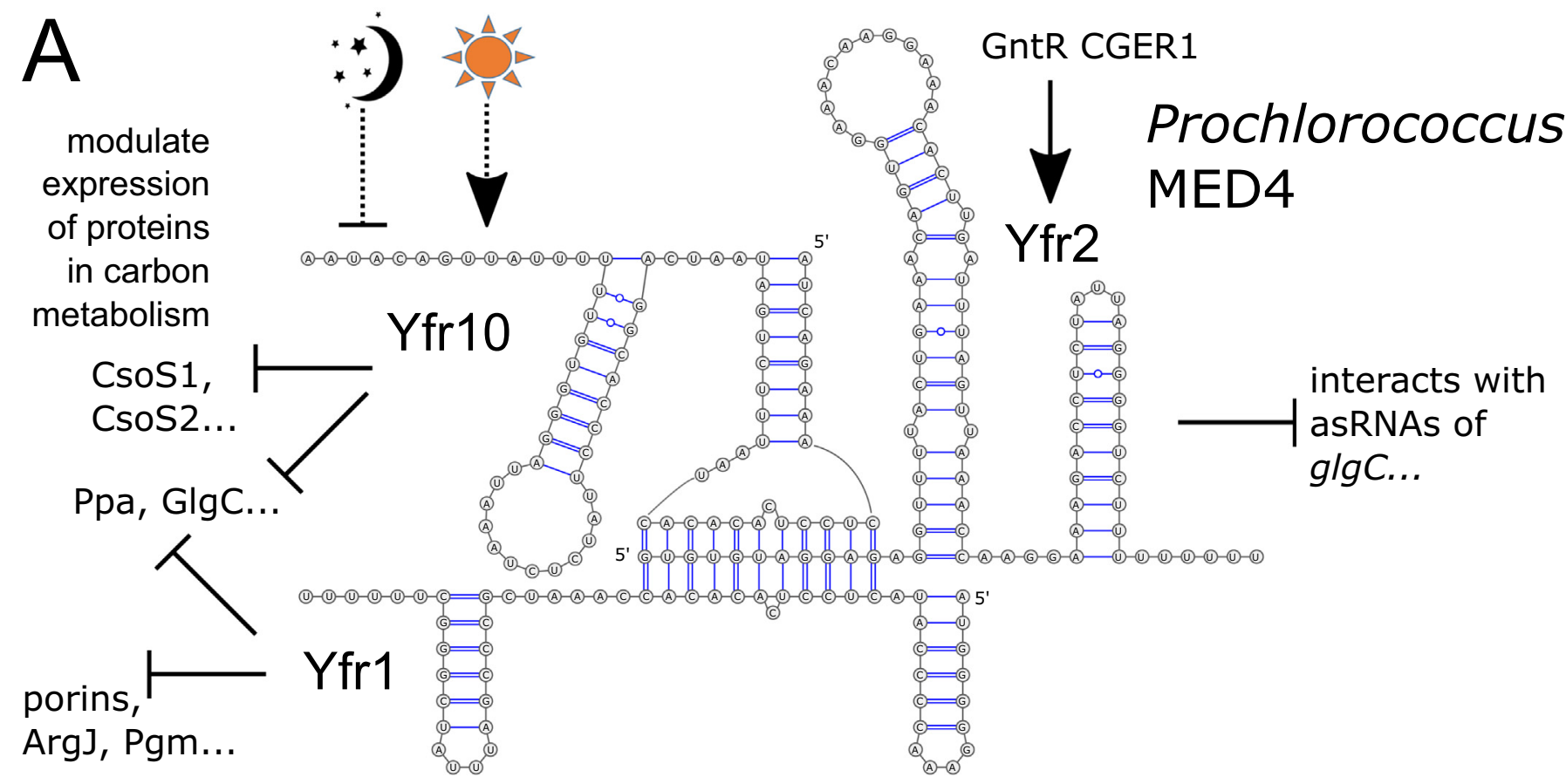

B

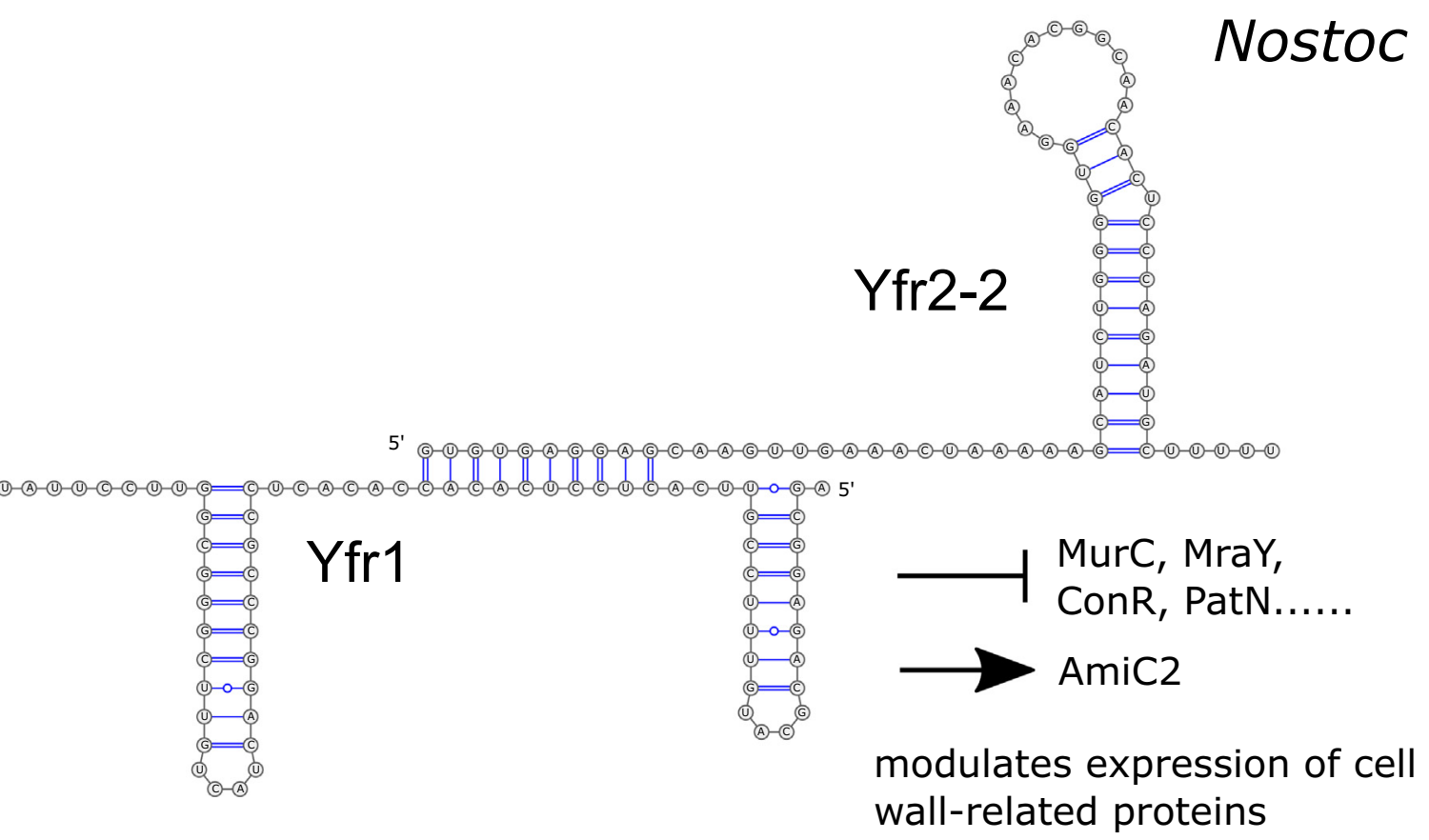

Fig. 2. The interplay between members of the Yfr1 and Yfr2 sRNA families as central element of a post-transcriptional regulatory mechanism.

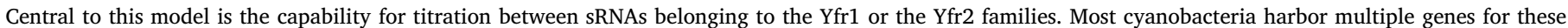

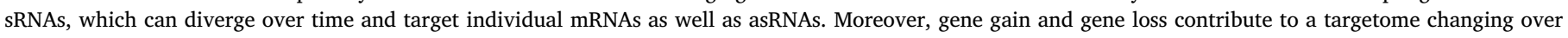

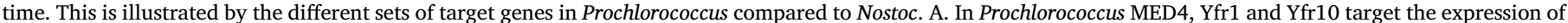

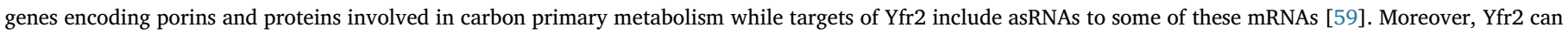

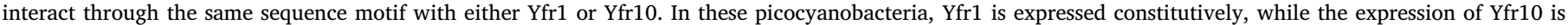

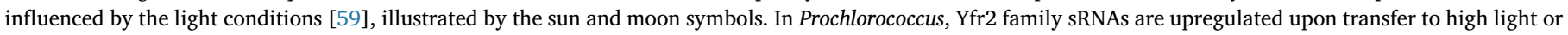

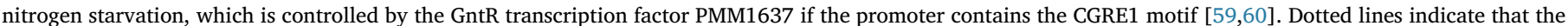

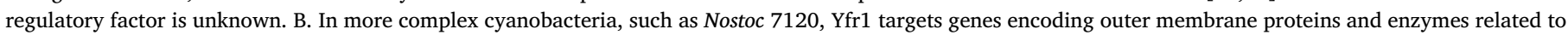
peptidoglycan biosynthesis and turnover [58].

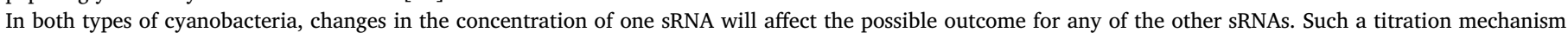

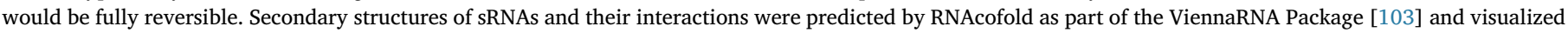
using VARNA [104]. Positive regulation is indicated by arrows, negative regulation by lines with blunt ends. 
homeostasis and correct cell wall remodeling during heterocysts differentiation [58].

These observations are reminiscent of the competing endogenous RNA (ceRNA) hypothesis in eukaryotic cells, which suggests that competition for a limited pool of regulatory RNAs mediates cross-regulation of mRNAs [61,62]. Evidence that a network of RNA crosstalk operates in bacteria has been discussed [63] and the Yfr1-Yfr2 system existing in cyanobacteria extends these suggestions by adding sets of sRNAs capable of mutually titrating each other.

\section{Light and iron: important factors for oxygenic photosynthesis}

The light quantity and quality and the concentration of bioavailable iron are of outstanding relevance for oxygenic photosynthesis and ultimately, the fixation of $\mathrm{C}_{\mathrm{i}}$ and therefore the $\mathrm{C} / \mathrm{N}$ balance. Several ncRNAs play an important role in the acclimation to changes in these conditions in cyanobacteria.

\subsection{Non-coding RNAs in the acclimation to low iron in cyanobacteria}

Regarding the acclimation to low iron, multiple asRNAs and sRNAs were found in Synechococcus WH 7803, Nostoc 7120, Synechocystis 6803 and Synechocystis 6714 that exhibited dramatic abundance changes when cultures were transferred to iron-deplete conditions $[40,41,64,65]$. However, only two of these asRNAs and a single sRNA have been studied at the molecular detail so far.

Expression of the gene for the ferric uptake regulator (furA), the transcription factor which governs the repression of many genes under iron-replete conditions, is covered by an asRNA from the extremely long 3' UTR of gene alr1690 in Nostoc 7120 [66]. Mutants lacking alr1690 and this asRNA developed an iron deficiency phenotype [67], suggesting functional relevance of this regulation. Analogous asRNAs to the furA gene were also found in the unicellular cyanobacteria Microcystis aeruginosa PCC 7806 and Synechocystis 6803 [68,69], supporting the possible relevance of these putative regulators further.

In both Synechocystis 6803 and Synechocystis 6714, the isiA gene encoding the iron stress-induced protein A is associated with an asRNA called IsrR. IsrR is transcribed from a constitutive, relatively weak promoter but accumulates under all conditions, except low iron, to a high level $[41,65]$. The function of IsrR is to prevent the premature expression of isiA by co-degradation in a threshold linear-response mechanism [70-72]. The tight relationship between isiA and its asRNA IsrR is further pronounced by the presence of an identical HLR1 ("high light regulatory 1") element in their promoters. The HLR1 element consists of a pair of imperfect 8-nt long direct repeats (G/T)TTACA(T/ A)(T/A) separated by two random nucleotides and constitutes the binding site for the transcription factor RpaB [73,74]. According to its relative location within the promoter, $\mathrm{RpaB}$ can repress or activate transcription (recently reviewed by Riediger et al. [75]). With regard to the isiA/IsrR system, the HLR1 element is in a repressing position in the promoter of the asRNA and in an activating position in the isiA gene promoter [76].

The 68 nt sRNA IsaR1 (iron stress-activated RNA1) was initially found in Synechocystis 6803, called NC-181 or Ncl1600 [40,65]. IsaR1 was later recognized to be widely conserved and to exist in unicellular and filamentous, freshwater, marine, symbiotic, mesophilic, or thermophilic cyanobacteria [77]. IsaR1 functions mainly as a repressor of gene expression during phases of low iron availability. IsaR1 is functionally highly relevant because its deletion in Synechocystis 6803 leads to a lethal phenotype under prolonged iron starvation [77]. Among its targets are mRNAs encoding electron carriers such as the major ferredoxin Fed1, cytochrome $c 6$ (PetJ) and the cytochrome $b_{6} f$ complex proteins PetABDC1 (Fig. 3). Furthermore, IsaR1 is a repressor of the iron sulfur cluster biogenesis proteins SufBCDS, the tetrapyrrole biosynthesis enzymes HemA and ChlN and two iron-containing enzymes, the superoxide dismutase subunit SodB and the aconitate hydratase
AcnB [77]. Homologs of the latter two targets are also recognized by RyhB, an sRNA in E. coli [78]. Moreover, similar to RyhB, whose transcription is repressed by the ferric uptake regulator (Fur) and therefore becomes de-repressed when $\mathrm{Fe}^{2+}$ concentrations are low, IsaR1 is under control of the cyanobacterial Fur homolog [77]. In $S y$ nechocystis 6803, Fur that is not bound to DNA is rapidly degraded by the heteromeric $\mathrm{FtsH} 1 / 3$ protease preventing re-binding and hence exerting an additional level of control $[79,80]$.

IsaR1 shows no sequence or structural similarity to RyhB or the homologs of functionally related sRNAs in other bacteria than cyanobacteria. Therefore, the regulation of iron homeostasis through sRNAs in very different groups of bacteria must be highly advantageous. With regard to cyanobacteria, the major target of IsaR 1 is the photosynthetic apparatus, which is impacted in multiple ways (Fig. 3).

Under natural conditions, microorganisms are rarely exposed to a single stress factor but multiple different stress factors may occur in parallel. Therefore it is interesting that, in addition to the mentioned iron-related genes, IsaR1 targets also the ggpS mRNA [81]. GgpS is the key enzyme for the catalysis of the compatible solute glucosylglycerol, which allows the cell to survive moderate increases in salinity. As a result, IsaR1 helps prioritizing the acclimation to low iron over the acclimation to increased osmolarity by delaying the increase in $g g p S$ expression [81].

\subsection{Changes in light intensity and the role of non-coding RNAs}

Light is the major source of energy for a photosynthetic organism. Therefore, changes in light intensity are sensed and can lead to dramatic changes in gene expression. There are several ncRNAs in cyanobacteria that play a role in the acclimation to different light conditions (Table 1). Some of these ncRNA were reviewed previously [53] and will be covered here only briefly.

There are three asRNAs in Synechocystis 6803, RblR, PsbA2R and PsbA3R, which play a protective role for their respective partners, the $r b c L, p s b A 2$ and psbA3 mRNAs [82,83], rather than participating in codegradation with their cognate mRNAs. In case of PsbA2R the protective function was studied in more detail and found to be due to shielding an RNase E-sensitive site, which led to a measurable increase in the psbA-encoded D1 protein content and photosynthetic capacity under HL conditions [82]. The function of As1-Flv4, to the contrary, is to prevent the premature expression of the flv4-2 operon after shift to LC via co-degradation [84].

Transcriptome analyses identified sRNAs with strongly increased expression levels after shifts to higher light intensities in several species (Table 1). Such sRNAs are candidates for regulators of photosynthesis because the photosynthetic machinery responds very sensitive to sudden increases in irradiance. However, only a single sRNA, PsrR1, has been characterized in detail thus far. PsrR1 is widely conserved in the cyanobacterial phylum. Its overexpression led in Synechocystis 6803 to a bleaching phenotype [39] indeed suggesting a photosynthesis-related function of PsrR1. The combined screening by experimental and computational methods led to the identification of several target genes, which mainly encode proteins with a role in photosynthesis, such as phycocyanin subunits, phycobilisome linker proteins, several photosystem I-related proteins, cytochrome $c 553$ and subunit $\mathrm{N}$ of the lightindependent protochlorophyllide reductase [85]. Following these results, the function of PsrR1 was established to down-regulate the expression of photosynthesis-related genes when cells are exposed to increased light intensities. To achieve this regulation, transcription of PsrR1 becomes upregulated at the higher light intensity. This upregulation is mediated by RpaB, which loses the capacity for DNA binding upon shift to HL, leading to a rapid de-repression of psrR1 transcription within a few minutes [86]. For Synechocystis 6803, the measured binding of RpaB to the psrR1 promoter DNA dropped from $65 \%$ to less than $20 \%$ already $5 \mathrm{~min}$ after transfer to $300 \mu \mathrm{mol}$ quanta $\mathrm{m}^{-2} \mathrm{~s}^{-1}$ [76]. The identification of $\mathrm{RpaB}$ as the major regulator of PsrR1 


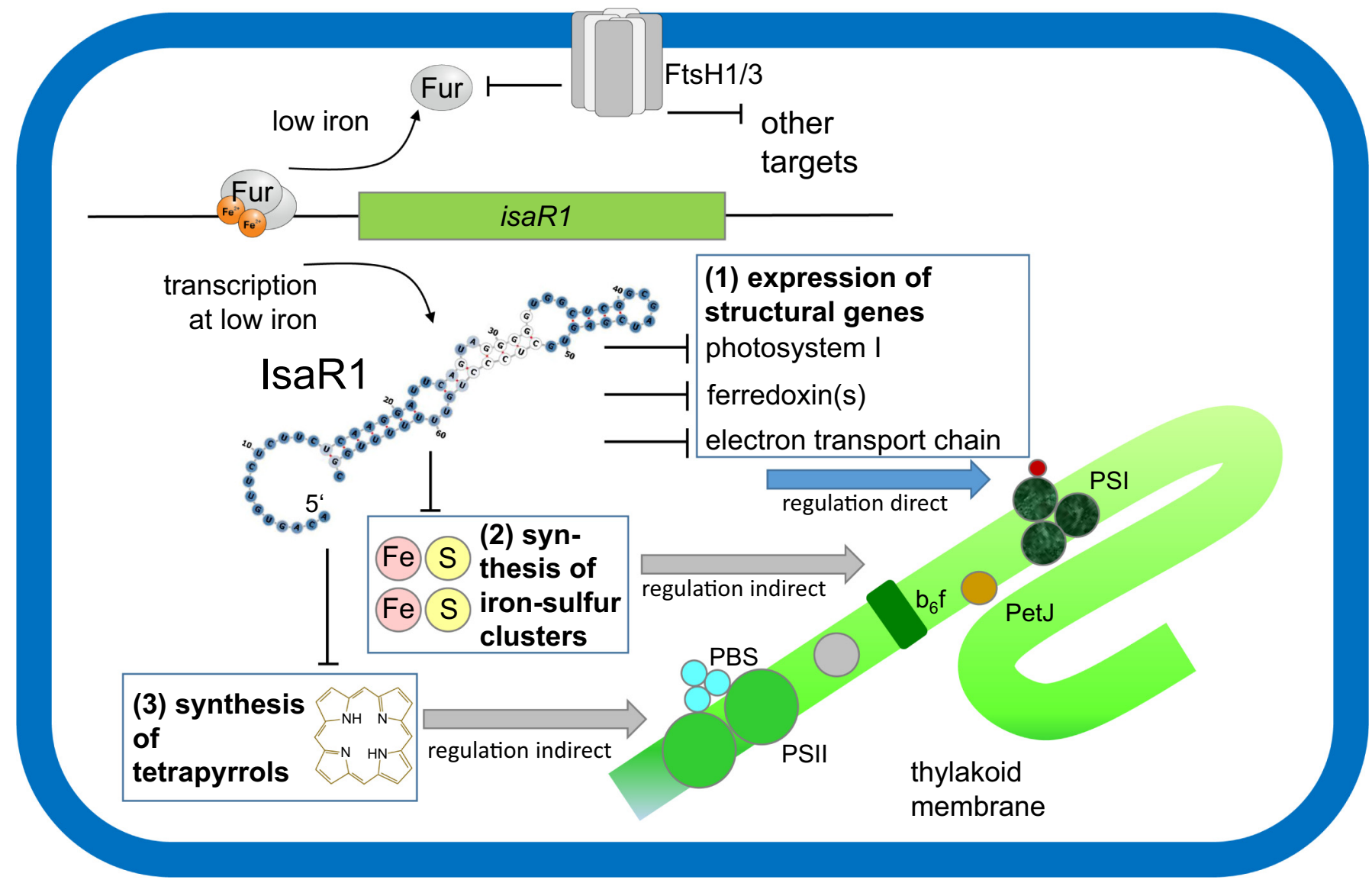

Fig. 3. IsaR1 and the photosynthetic membrane. Under low iron conditions, IsaR1 is controlling the photosynthetic machinery in several different ways. (1) IsaR1 directly represses the translation and stability of mRNAs encoding proteins involved in photosynthesis such as ferredoxin, cytochrome $c 6$ and the cytochrome $b_{6} f$ complex; (2) IsaR1 controls the only and essential pathway for the biosynthesis of iron sulfur clusters in Synechocystis 6803; (3) IsaR1 represses hemA encoding glutamyl-tRNA reductase, the first committed enzyme in the C5 pathway, the only and essential pathway for the production of tetrapyrroles (chlorophylls, heme groups and linear tetrapyrroles), while chlorophyll synthesis is affected further through another two targets, chlN and chlH [77]. The Ferric uptake regulator Fur is depicted either with $\mathrm{Fe}^{2+}$, binding to DNA or without, not binding to DNA. In the unbound state, Fur is rapidly degraded by the cell membrane-associated FtsH1/3 protease preventing re-binding [80]. The FtsH1/3 protease also affects expression of the NdhR-, the Pho- and the NtcA-regulons during phases of limitations in the respective nutrients $C_{i}$, phosphate or nitrogen [80], indicated by "other targets". Hence, the FtsH1/3 protease complex exerts an additional level of control in the acclimation responses to these stress situations.

transcription revealed a particular feature in the action of this sRNA. $\mathrm{RpaB}$ acts as an important transcription factor, enhancing the transcription of many PSI genes under low light (recently summarized by Riediger et al. [75]), with a large overlap in genes also targeted by PsrR1. This points to the dual repression of PSI genes after shifts to HL at the transcriptional level by RpaB because it becomes rapidly inactive and at the post-transcriptional level through repression by PsrR1 [85], together constituting a feed-forward loop in the regulation of these genes.

Table 1

Selected ncRNAs in cyanobacteria with a characterized role in the acclimation to different light conditions or changes in $\mathrm{C}_{\mathrm{i}}$ supply. The name of the respective ncRNA is given, followed by information on whether it is an sRNA or asRNA, the length of detected major bands in nt, the strain in which the characterization primarily was carried out, the condition under which strong regulation was observed (LC, low carbon; HL, high light; DK, darkness) and the direction of this regulation. Finally, the function is briefly mentioned, together with the level of evidence (E, experimental; P, prediction only) and the respective publication.

\begin{tabular}{|c|c|c|c|c|c|c|}
\hline Name & Type & Length & Species & Regulation & Function & Ref. \\
\hline As1-Flv4 & asRNA & 500,280 & Synechocystis sp. PCC 6803 & LC down & Preventing premature expression of the flv4-2 operon after shift to LC (E) & [84] \\
\hline PsbA2R & asRNA & 130,220 & Synechocystis sp. PCC 6803 & HL up & Protecting psbA2 mRNA from premature degradation (E) & [82] \\
\hline PsbA3R & asRNA & 160,180 & Synechocystis sp. PCC 6803 & HL up & Protecting psbA3 mRNA from premature degradation (E) & [82] \\
\hline RblR & asRNA & 113 & Synechocystis sp. PCC 6803 & Unknown & Activating $r b c L$ expression (E) & [83] \\
\hline PsrR1 & sRNA & 131 & Synechocystis sp. PCC 6803 & HL up & $\begin{array}{l}\text { Limiting expression of photosynthesis-related genes upon shift to HL, psaL, psaJ, chlN, } \\
p s b B, c p c A \text { and several others (E) }\end{array}$ & [85] \\
\hline PmgR1 & sRNA & 251 & Synechocystis sp. PCC 6803 & DK up; LC up & Required for photo-mixotrophic growth and regulation of glycogen accumulation (E) & [101] \\
\hline Sye_sRNA3 & sRNA & 500 & S. elongatus UTEX 2973 & DK up & Unknown & [42] \\
\hline Yfr20 & sRNA & 89 & Prochlorococcus MED4 & HL up & Unknown & {$[56]$} \\
\hline $\mathrm{i} 403$ & sRNA & 60 & Synechococcus sp. WH 7803 & HL up & cpeB, mpeB (P) & [64] \\
\hline $\mathrm{i} 812$ & sRNA & 60 & Synechococcus sp. WH 7803 & HL up & cpeB, mpeB, cpeE, petA (P) & [64] \\
\hline
\end{tabular}




\section{Riboregulatory controls in nitrogen and carbon metabolism in cyanobacteria}

This section covers N-regulated ncRNAs in different cyanobacteria except those in multicellular species that were assigned to the HetR regulon and therefore might be potentially involved in the differentiation and/or the function of heterocysts. Those are reviewed in chapter 5 below.

Global analyses of the N-responsive transcriptome allowed the identification of N-regulated ncRNAs both in unicellular and filamentous strains of cyanobacteria $[40,41,43,56]$. Similar to observations made in the case of other CRP-family transcription factors, the NtcA regulon includes $\mathrm{N}$-regulated sRNAs and asRNAs. These sRNAs were designated Nitrogen stress induced RNAs (NsiR) or Nitrogen stress repressed RNAs (NsrR) depending on the direction of regulation $[38,43,51]$.

Using ChIP-Seq as an alternative approach, Giner-Lamia et al. characterized the NtcA regulon in Synechocystis 6803 [87]. In their study, 18 sRNAs and 5 asRNAs with assigned NtcA binding sites in their respective promoters were identified. The combined results from these studies demonstrated that there is a considerably understudied set of ncRNA regulators potentially involved in the control of nitrogen metabolism. A particularly interesting candidate is the sRNA NsiR7 (Ncr0210) in Synechocystis 6803, which was experimentally validated to be under NtcA control [87] and which is also induced under conditions of carbon limitation and is likely co-regulated by NdhR $[88,89]$. Thus, NsiR7 likely plays an integrative role related to the $\mathrm{C} / \mathrm{N}$ status but the possible targets have not been analyzed so far.

Two NtcA-regulated sRNAs, NsrR1 [90] and NsiR4 [91] have been further characterized (Fig. 4). Transcription of NsrR1 (nitrogen stress repressed RNA1) is regulated in response to nitrogen availability in Nostoc 7120, and its repression under nitrogen limitation is operated, at least partially, by binding of NtcA to the promoter of nsrR1 [90]. Sequences encoding NsrR1 homologs are found in the genomes of heterocystous cyanobacteria and some unicellular strains that are phylogenetically close, but not in more distantly related cyanobacteria, suggesting its function might be linked to some specific trait of this group of cyanobacteria. Prediction of possible targets of NsrR1 regulation pointed to $n b l A$, encoding a protease adaptor involved in degradation of the cyanobacterial antenna complexes, called phycobilisomes, in response to different stresses. Interaction of NsrR1 with the $5^{\prime}$ UTR of $n b l A$ leads to increased degradation of the $n b l A$ mRNA and therefore modulates phycobilisome degradation in response to nitrogen availability. Therefore, NsrR1 participates, together with NblA, whose expression is positively regulated by NtcA, in a coherent feed-forward regulatory loop. Transcription of NsrR1 under nitrogen sufficient conditions sets a threshold for NblA expression and ensures that the accumulation of NblA, and therefore the degradation of phycobilisomes is not triggered by fluctuating nitrogen availability unless nitrogen limitation is established [90].

It was demonstrated both in Synechocystis 6803 and Nostoc 7120 that the sRNA NsiR4 is strongly upregulated under nitrogen-deplete conditions and that this upregulation is mediated by NtcA [91]. Homologs of NsiR4 exist in distantly related cyanobacteria. Two genes were identified as targets of NsiR4 in Synechocystis 6803, gifA (ssl1911) encoding the glutamine synthetase inactivating factor IF7 and $s s r 1528$ encoding a DUF4090 domain-containing small protein of unknown function [91]. The expression of both genes is repressed under nitrogen depletion consistent with their negative regulation by NtcA. IF7 is one of two inhibitory factors that lead to the inactivation of the enzyme glutamine synthetase when nitrogen supply exceeds demand [92]. However, the second factor, called IF17, encoded by the gifB gene and considered the physiologically more relevant inhibitor of glutamine synthetase activity, is not controlled by NsiR4. Nonetheless, expression

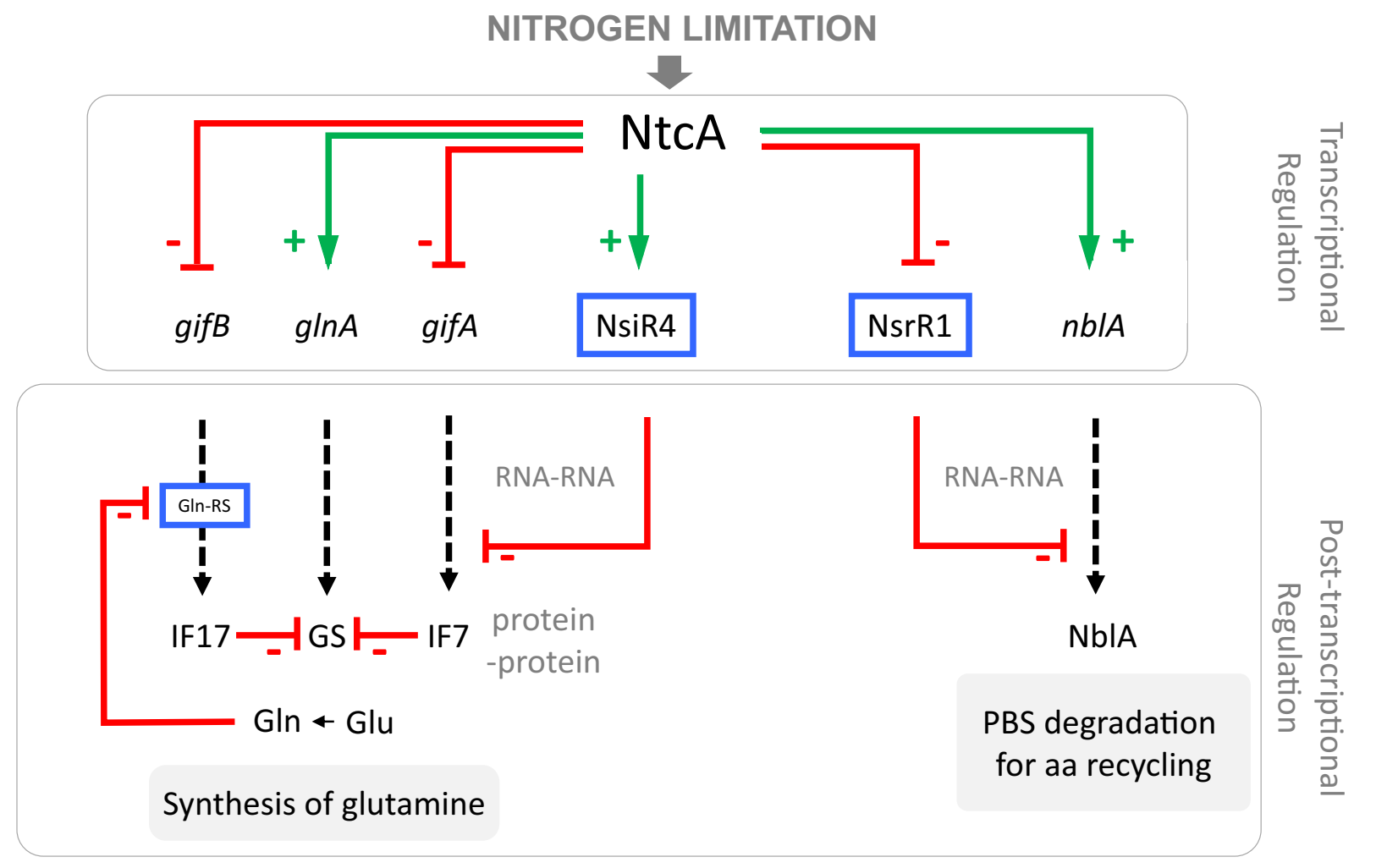

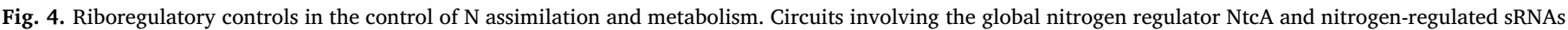

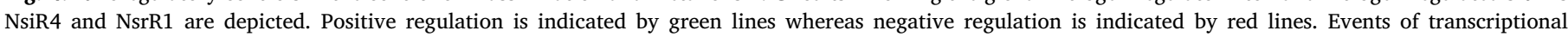

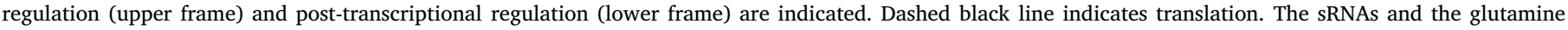

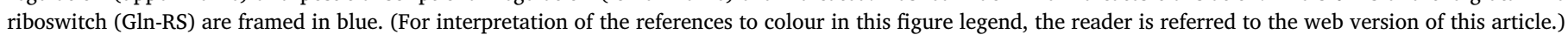


of the gifB gene was found to be under riboregulatory control, but through a fundamentally distinct mechanism. Based on previous work that had characterized the $\sim 60 \mathrm{nt}$ long so-called $\operatorname{gln} A$ RNA motif of Synechococcus elongatus and the downstream peptide (DP) motif of Synechococcus sp. CC9902 as glutamine-binding aptamers [93], a homologous element was identified and characterized as a riboswitch within the gifB 5'UTR [35]. Both types of aptamers have some similarity but the $g \ln A$ element consists of a three-way helical junction with a loop $\mathrm{E}$ motif, while the other contains a pseudoknot. Klähn et al. suggested to rename the $g \ln A$ and DP riboswitches to glutamine type 1 and glutamine type 2 riboswitches, respectively [35]. Using a GFP fusion, it was demonstrated that the glutamine type 1 riboswitch acts as an ON switch to control the synthesis of IF17 [35]. The structure of the glutamine type 2 riboswitch ligand binding domain has recently been solved by Xray crystallography revealing three coaxial helical domains, from which the central one contains the pseudoknot, which exhibited partial triplex character [94]. These findings showed that glutamine is indeed a signaling molecule in cyanobacteria and that, in contrast to any other bacterial phylum investigated thus far, the glutamine level is specifically sensed by an RNA element. This sensing establishes a feedback mechanism by inducing the accumulation of the major GS inhibitor IF17, which in turn inactivates GS and leads to a rapid drop in glutamine synthesis (Fig. 4).

Hence, cyanobacteria evolved efficient and unique mechanisms to control $\mathrm{N}$ assimilation that differ considerably from the archetypical model developed for $E$. coli and utilize, with NsiR4 and the glutamine riboswitch, two different riboregulatory components.

\section{Regulatory RNA in the control of nitrogen fixation and heterocyst differentiation}

Several groups of cyanobacteria are able to carry out nitrogen fixation, which, due to the extreme sensitivity of the enzyme nitrogenase to $\mathrm{O}_{2}$, requires sophisticated strategies to avoid damage by photosynthetically produced $\mathrm{O}_{2}$. One such strategy is the differentiation, in a semi-regular pattern along the filaments, of certain cells, the heterocysts, devoted to nitrogen fixation. In addition to morphological changes that reduce the entry of gases, the metabolism of the vegetative cells that differentiate as heterocysts must be completely transformed to meet the needs of a nitrogen-fixing factory. During differentiation, respiration is enhanced, photosynthetic $\mathrm{CO}_{2}$ fixation and $\mathrm{O}_{2}$ production stops and the mature heterocyst becomes heterotrophic, since it relies on carbon-containing molecules imported from adjacent vegetative cells. These metabolic adjustments require precise regulation of gene expression, presumably both at the transcriptional and the post-transcriptional levels.

Transcriptional control of the development of heterocysts has recently been reviewed and depends both on the global nitrogen control regulator NtcA and the regulator of cellular differentiation HetR [95]. Expression of both genes is mutually dependent and is increased in cells becoming heterocysts [96]. HetR-dependent changes in the abundance of every transcript generated from the whole genome of Nostoc sp. PCC 7120 , have been analyzed [43,97]. The HetR-dependent transcriptome includes, in addition to protein-coding genes, several sRNAs, such as NsiR1 [38], NsiR2 [43], NsiR8 and NsiR9 [51], as well as transcripts annotated as asRNAs. Post-transcriptional regulation exerted by these heterocyst-specific transcripts could contribute to the metabolic features of these specialized cells.

NsiR1, the first nitrogen-regulated sRNA described in cyanobacteria, was identified in Nostoc 7120 as a 60 nt molecule transcribed from a region that included an array of predicted transcriptional terminators, each corresponding to one copy of the nsiR1 gene [38]. Expression of NsiR1 requires HetR and takes place from promoters containing the DIF1 motif found associated to very early expression specifically in cells that are becoming heterocysts $[43,98]$. The observation that NsiR1 is transcribed from several copies, each of them bearing heterocyst- specific promoters, suggests that a strong differential expression of NsiR1 takes place in heterocysts. Interestingly, one of the copies of $n s i R 1$ is located antisense to the 5'UTR of the hetF gene required for heterocyst differentiation, raising the possibility of a post-transcriptional regulation of hetF by NsiR1. This arrangement is conserved across genomes of heterocystous cyanobacteria.

Similar to NsiR1, three sRNAs that are encoded in the genomes from several heterocystous strains, NsiR2, NsiR8 and NsiR9, also appear transcriptionally co-expressed with previously known heterocyst-specific genes, and in fact NsiR2 and NsiR8 are transcribed from promoters bearing the recently described DIF2 motif, which, similar to DIF1 is associated to heterocyst-specific transcription during initial steps of differentiation. In contrast, expression of NsiR9 takes place at later time points during heterocyst differentiation [97]. Although no function has been described for these sRNAs so far, the observation that they are conserved in several cyanobacterial genomes and transcribed from HetR-dependent, heterocyst-specific, promoters with conserved sequence motifs strongly suggests these transcripts might be exerting some type of yet unknown post-transcriptional regulation specifically in differentiating cells.

Several asRNAs have been described in the HetR regulon that are transcriptionally co-expressed with previously described genes involved in early aspects of the morphological and metabolic transformation of vegetative cells into heterocysts [97]. The function of one asRNA could be clarified in this context (Fig. 5). Transcription of as $g l p X$ takes place specifically in heterocysts from a promoter containing a DIF1 motif. The interaction between as_glpX and the $g l p X$ mRNA encoding the CalvinBenson-Bassham cycle enzyme sedoheptulose-1,7-bisphosphatase leads to RNase III-mediated degradation of the duplex, therefore contributing to downregulating the levels of this enzyme and to a reduction of the photosynthetic capacity specifically in the (pro)heterocysts that produce as.glpX. The regulation operated by a heterocyst-specific asRNA would thus contribute to the extensive metabolic reprogramming that takes place during maturation of this specialized cell type.

\section{Concluding remarks}

Accumulating evidences point to abundant non-coding transcription in all bacteria analyzed. Although some ncRNAs described in this review have been analyzed in detail, the vast majority of likely relevant ncRNAs are still uncharacterized, also in model cyanobacteria. However, both their phylogenetic conservation across genomes of relatively distant cyanobacteria and their regulated transcription in response to some major stresses for these photosynthetic bacteria (including light shifts, iron, nitrogen or $\mathrm{C}_{\mathrm{i}}$ availability) suggests that some of these ncRNAs may be of regulatory relevance.

Similar to the observations made for major transcription factors in other bacteria, whose regulons have been analyzed in detail, ncRNAs are found among the transcripts regulated by NtcA (global nitrogen regulator in cyanobacteria) or $\mathrm{RpaB}$ (operating regulation in response to light shifts). The interplay between a transcription factor such as NtcA, Fur or RpaB and some type of regulatory RNA (Fig. 4), and the resulting mixed circuits yield unique regulatory features, including different forms of feed-forward loops and feedback loops and thus are fundamental architectural elements of regulatory networks [99,100].

To summarize, recent findings in cyanobacteria have demonstrated the existence of versatile riboregulatory mechanisms which are involved in keeping the $\mathrm{C} / \mathrm{N}$ balance in check. It can be assumed that analogous mechanisms likely exist for other photosynthetic organisms as well. Even more so surprising is the total lack of such information for plants, hence encouraging a view that the search for appropriate miRNAs and other types of ncRNA regulators should be extended.

\section{Declaration of competing interest}

The authors declare that they have no known competing financial 


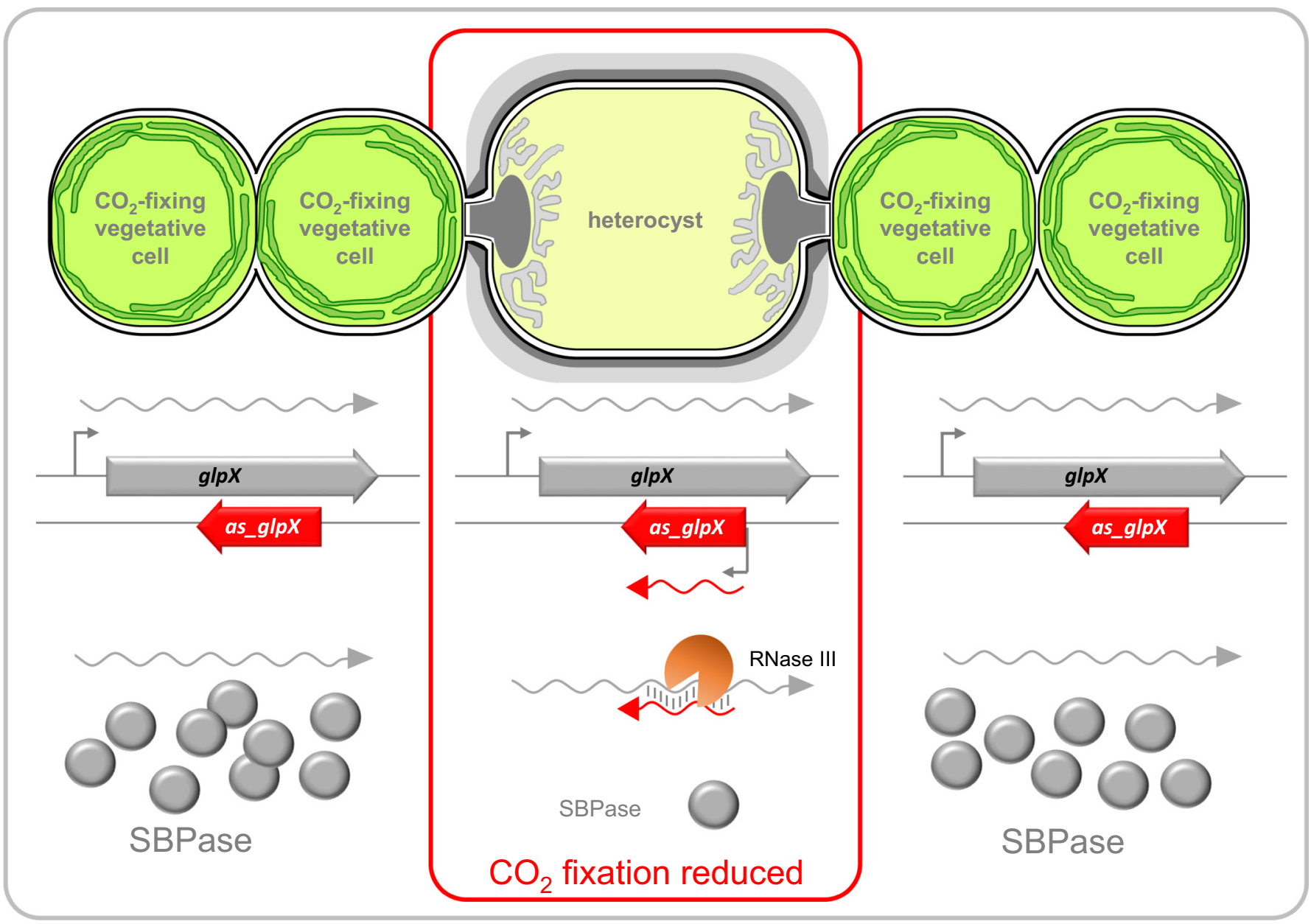

Fig. 5. The function of a heterocyst-specific asRNA to the $g l p X$ gene in the context of heterocyst differentiation. In vegetative cells, as $g l p X$ is not transcribed, while in developing heterocysts the transcription of $a s_{s} g l p X$ (red arrow) promotes the cleavage of $g l p X$ mRNA (grey arrow) by RNase III, resulting in the reduction of SBPase and therefore photosynthetic capacity in these specialized cells. (For interpretation of the references to colour in this figure legend, the reader is referred to the web version of this article.)

interests or personal relationships that could have appeared to influence the work reported in this paper.

\section{Acknowledgements}

Our work on novel cyanobacterial RNA regulators and their participation in regulatory networks has been supported by the German Research Foundation, grant HE 2544/15-1 "SCyCode" to WRH and by BFU2016-74943-C2-1-P (AEI/FEDER, EU) to AMMP.

\section{References}

[1] P. Flombaum, J.L. Gallegos, R.A. Gordillo, J. Rincón, L.L. Zabala, N. Jiao, D.M. Karl, W.K.W. Li, M.W. Lomas, D. Veneziano, C.S. Vera, J.A. Vrugt, A.C. Martiny, Present and future global distributions of the marine Cyanobacteria Prochlorococcus and Synechococcus, Proc. Natl. Acad. Sci. U. S. A. 110 (2013) 9824-9829.

[2] F. Partensky, W.R. Hess, D. Vaulot, Prochlorococcus, a marine photosynthetic prokaryote of global significance, Microbiol. Mol. Biol. Rev. 63 (1999) 106-127.

[3] M. Calvin, The path of carbon in photosynthesis, Science 135 (1962) 879-889.

[4] F.E. Allison, H.J. Morris, Nitrogen fixation by blue-green algae, Science 71 (1930) $221-223$.

[5] E.P. Suosaari, R.P. Reid, P.E. Playford, J.S. Foster, J.F. Stolz, G. Casaburi, P.D. Hagan, V. Chirayath, I.G. Macintyre, N.J. Planavsky, G.P. Eberli, New multiscale perspectives on the stromatolites of Shark Bay, Western Australia, Sci. Rep. 6 (2016) 20557.

[6] H. Bolhuis, L. Fillinger, L.J. Stal, Coastal microbial mat diversity along a natural salinity gradient, PLoS One 8 (2013) e63166.

[7] M. Hagemann, M. Henneberg, V.J.M.N.L. Felde, S.M. Berkowicz, H. Raanan, N. Pade, P. Felix-Henningsen, A. Kaplan, Cyanobacterial populations in biological soil crusts of the Northwest Negev Desert, Israel - effects of local conditions and disturbance, FEMS Microbiol. Ecol. (2016) 93.

[8] C.C. Gaylarde, P.M. Gaylarde, B.A. Neilan, Endolithic phototrophs in built and natural stone, Curr. Microbiol. 65 (2012) 183-188.

[9] C. Johansson, B. Bergman, Early events during the establishment of the Gunnera Nostoc symbiosis, Planta 188 (1992) 403-413.

[10] L. Ran, J. Larsson, T. Vigil-Stenman, J.A.A. Nylander, K. Ininbergs, W.-W. Zheng, A. Lapidus, S. Lowry, R. Haselkorn, B. Bergman, Genome erosion in a nitrogenfixing vertically transmitted endosymbiotic multicellular cyanobacterium, PLoS One 5 (2010) e11486.

[11] E.C.M. Nowack, M. Melkonian, G. Glöckner, Chromatophore genome sequence of Paulinella sheds light on acquisition of photosynthesis by eukaryotes, Curr. Biol. 18 (2008) 410-418.

[12] A.W. Thompson, R.A. Foster, A. Krupke, B.J. Carter, N. Musat, D. Vaulot, M.M.M. Kuypers, J.P. Zehr, Unicellular cyanobacterium symbiotic with a singlecelled eukaryotic alga, Science 337 (2012) 1546-1550.

[13] J.A. Hilton, R.A. Foster, H. James Tripp, B.J. Carter, J.P. Zehr, T.A. Villareal, Genomic deletions disrupt nitrogen metabolism pathways of a cyanobacterial diatom symbiont, Nat. Commun. 4 (2013) 1767.

[14] T. Nakayama, M. Nomura, Y. Takano, G. Tanifuji, K. Shiba, K. Inaba, Y. Inagaki, M. Kawata, Single-cell genomics unveiled a cryptic cyanobacterial lineage with a worldwide distribution hidden by a dinoflagellate host, Proc. Natl. Acad. Sci. U. S. A. 116 (2019) 15973-15978.

[15] D. Bombar, P. Heller, P. Sanchez-Baracaldo, B.J. Carter, J.P. Zehr, Comparative genomics reveals surprising divergence of two closely related strains of uncultivated UCYN-A cyanobacteria, ISME J 8 (2014) 2530-2542.

[16] T. Dagan, M. Roettger, K. Stucken, G. Landan, R. Koch, P. Major, S.B. Gould, V.V. Goremykin, R. Rippka, N. Tandeau de Marsac, M. Gugger, P.J. Lockhart, J.F. Allen, I. Brune, I. Maus, A. Pühler, W.F. Martin, Genomes of Stigonematalean cyanobacteria (subsection $\mathrm{V}$ ) and the evolution of oxygenic photosynthesis from prokaryotes to plastids, Genome Biol Evol 5 (2013) 31-44, https://doi.org/10. 1093/gbe/evs117.

[17] L.A. Finney, T.V. O'Halloran, Transition metal speciation in the cell: insights from the chemistry of metal ion receptors, Science 300 (2003) 931-936. 
[18] N. Keren, R. Aurora, H.B. Pakrasi, Critical roles of bacterioferritins in iron storage and proliferation of cyanobacteria, Plant Physiol. 135 (2004) 1666-1673.

[19] M. Behrenfeld, Z.S. Kolber, Widespread iron limitation of phytoplankton in the South Pacific Ocean, Science 283 (1999) 840-843.

[20] A. Herrero, E. Flores, Genetic responses to carbon and nitrogen availability in Anabaena, Environ. Microbiol. 21 (2019) 1-17.

[21] M.I. Muro-Pastor, J.C. Reyes, F.J. Florencio, Cyanobacteria perceive nitrogen status by sensing intracellular 2-oxoglutarate levels, J. Biol. Chem. 276 (2001) 38320-38328.

[22] K. Forchhammer, Global carbon/nitrogen control by $\mathrm{P}_{\text {II }}$ signal transduction in cyanobacteria: from signals to targets, FEMS Microbiol. Rev. 28 (2004) 319-333.

[23] M.F. Vázquez-Bermúdez, A. Herrero, E. Flores, 2-Oxoglutarate increases the binding affinity of the NtcA (nitrogen control) transcription factor for the Synechococcus glnA promoter, FEBS Lett. 512 (2002) 71-74.

[24] R. Tanigawa, M. Shirokane, S. Maeda Si, T. Omata, K. Tanaka, H. Takahashi, Transcriptional activation of NtcA-dependent promoters of Synechococcus sp. PCC 7942 by 2 -oxoglutarate in vitro, Proc. Natl. Acad. Sci. U. S. A. 99 (2002) 4251-4255.

[25] I. Luque, M.F. Vázquez-Bermúdez, J. Paz-Yepes, E. Flores, A. Herrero, In vivo activity of the nitrogen control transcription factor NtcA is subjected to metabolic regulation in Synechococcus sp. strain PCC 7942, FEMS Microbiol. Lett. 236 (2004) $47-52$.

[26] R. Schwarz, K. Forchhammer, Acclimation of unicellular cyanobacteria to macronutrient deficiency: emergence of a complex network of cellular responses, Microbiology 151 (2005) 2503-2514.

[27] J. Espinosa, F. Rodríguez-Mateos, P. Salinas, V.F. Lanza, R. Dixon, F. de la Cruz, A. Contreras, PipX, the coactivator of NtcA, is a global regulator in cyanobacteria, Proc. Natl. Acad. Sci. U. S. A. 111 (2014) E2423-E2430.

[28] J. Espinosa, K. Forchhammer, S. Burillo, A. Contreras, Interaction network in cyanobacterial nitrogen regulation: PipX, a protein that interacts in a 2-oxoglutarate dependent manner with PII and NtcA, Mol. Microbiol. 61 (2006) 457-469.

[29] B. Watzer, P. Spät, N. Neumann, M. Koch, R. Sobotka, B. Macek, O. Hennrich, K. Forchhammer, The signal transduction protein PII controls ammonium, nitrate and urea uptake in cyanobacteria, Front. Microbiol. 10 (2019) 1428.

[30] K.A. Selim, F. Haase, M.D. Hartmann, M. Hagemann, K. Forchhammer, PII-like signaling protein SbtB links cAMP sensing with cyanobacterial inorganic carbon response, Proc. Natl. Acad. Sci. U. S. A. 115 (2018) E4861-E4869.

[31] S.M.E. Daley, A.D. Kappell, M.J. Carrick, R.L. Burnap, Regulation of the cyanobacterial $\mathrm{CO}_{2}$-concentrating mechanism involves internal sensing of $\mathrm{NADP}^{+}$and a-ketogutarate levels by transcription factor CcmR, PLoS One 7 (2012) e41286.

[32] T. Nishimura, Y. Takahashi, O. Yamaguchi, H. Suzuki, S.-I. Maeda, T. Omata, Mechanism of low $\mathrm{CO}_{2}$-induced activation of the cmp bicarbonate transporter operon by a LysR family protein in the cyanobacterium Synechococcus elongatus strain PCC 7942, Mol. Microbiol. 68 (2008) 98-109.

[33] Y.-L. Jiang, X.-P. Wang, H. Sun, S.-J. Han, W.-F. Li, N. Cui, G.-M. Lin, J.-Y. Zhang, W. Cheng, D.-D. Cao, Z.-Y. Zhang, C.-C. Zhang, Y. Chen, C.-Z. Zhou, Coordinating carbon and nitrogen metabolic signaling through the cyanobacterial global repressor NdhR, Proc. Natl. Acad. Sci. U. S. A. 115 (2018) 403-408.

[34] C.-C. Zhang, C.-Z. Zhou, R.L. Burnap, L. Peng, Carbon/nitrogen metabolic balance: lessons from cyanobacteria, Trends Plant Sci. 23 (2018) 1116-1130.

[35] S. Klähn, P. Bolay, P.R. Wright, R.M. Atilho, K.I. Brewer, M. Hagemann, R.R. Breaker, W.R. Hess, A glutamine riboswitch is a key element for the regulation of glutamine synthetase in cyanobacteria, Nucleic Acids Res. 46 (2018) 10082-10094.

[36] I.M. Axmann, P. Kensche, J. Vogel, S. Kohl, H. Herzel, W.R. Hess, Identification of cyanobacterial non-coding RNAs by comparative genome analysis, Genome Biol. 6 (2005) R73.

[37] B. Voss, G. Gierga, I.M. Axmann, W.R. Hess, A motif-based search in bacterial genomes identifies the ortholog of the small RNA Yfr1 in all lineages of cyanobacteria, BMC Genomics 8 (2007) 375.

[38] D. Ionescu, B. Voss, A. Oren, W.R. Hess, A.M. Muro-Pastor, Heterocyst-specific transcription of NsiR1, a non-coding RNA encoded in a tandem array of direct repeats in cyanobacteria, J. Mol. Biol. 398 (2010) 177-188.

[39] J. Mitschke, J. Georg, I. Scholz, C.M. Sharma, D. Dienst, J. Bantscheff, B. Voß, C. Steglich, A. Wilde, J. Vogel, W.R. Hess, An experimentally anchored map of transcriptional start sites in the model cyanobacterium Synechocystis sp. PCC6803, Proc. Natl. Acad. Sci. U. S. A. 108 (2011) 2124-2129.

[40] M. Kopf, S. Klähn, I. Scholz, J.K.F. Matthiessen, W.R. Hess, B. Voß, Comparative analysis of the primary transcriptome of Synechocystis sp. PCC 6803, DNA Res. 21 (2014) 527-539.

[41] M. Kopf, S. Klähn, I. Scholz, W.R. Hess, B. Voß, Variations in the non-coding transcriptome as a driver of inter-strain divergence and physiological adaptation in bacteria, Sci. Rep. 5 (2015) 9560.

[42] X. Tan, S. Hou, K. Song, J. Georg, S. Klähn, X. Lu, W.R. Hess, The primary transcriptome of the fast-growing cyanobacterium Synechococcus elongatus UTEX 2973, Biotechnol Biofuels 11 (2018) 218.

[43] J. Mitschke, A. Vioque, F. Haas, W.R. Hess, A.M. Muro-Pastor, Dynamics of transcriptional start site selection during nitrogen stress-induced cell differentiation in Anabaena sp. PCC7120, Proc. Natl. Acad. Sci. U. S. A. 108 (2011) 20130-20135.

[44] B. Voss, H. Bolhuis, D.P. Fewer, M. Kopf, F. Möke, F. Haas, R. El-Shehawy, P. Hayes, B. Bergman, K. Sivonen, E. Dittmann, D.J. Scanlan, M. Hagemann, L.J. Stal, W.R. Hess, Insights into the physiology and ecology of the brackish water-adapted cyanobacterium Nodularia spumigena CCY9414 based on a genometranscriptome analysis, PLoS One 8 (2013) e60224.

[45] M. Kopf, F. Möke, H. Bauwe, W.R. Hess, M. Hagemann, Expression profiling of the bloom-forming cyanobacterium Nodularia CCY9414 under light and oxidative stress conditions, ISME J 9 (2015) 2139-2152.

[46] U. Pfreundt, M. Kopf, N. Belkin, I. Berman-Frank, W.R. Hess, The primary transcriptome of the marine diazotroph Trichodesmium erythraeum IMS101, Sci. Rep. 4 (2014) 6187.

[47] N. Walworth, U. Pfreundt, W.C. Nelson, T. Mincer, J.F. Heidelberg, F. Fu, J.B. Waterbury, T. Glavina del Rio, L. Goodwin, N.C. Kyrpides, M.L. Land, T. Woyke, D.A. Hutchins, W.R. Hess, E.A. Webb, Trichodesmium genome maintains abundant, widespread noncoding DNA in situ, despite oligotrophic lifestyle, Proc. Natl. Acad. Sci. U. S. A. 112 (2015) 4251-4256.

[48] D. Baumgartner, M. Kopf, S. Klähn, C. Steglich, W.R. Hess, Small proteins in cyanobacteria provide a paradigm for the functional analysis of the bacterial microproteome, BMC Microbiol. 16 (2016) 285.

[49] I.M. Axmann, J. Holtzendorff, B. Voss, P. Kensche, W.R. Hess, Two distinct types of 6S RNA in Prochlorococcus, Gene 406 (2007) 69-78.

[50] G. Gierga, B. Voss, W.R. Hess, The Yfr2 ncRNA family, a group of abundant RNA molecules widely conserved in cyanobacteria, RNA Biol. (2009) 222-227.

[51] M. Brenes-Álvarez, E. Olmedo-Verd, A. Vioque, A.M. Muro-Pastor, Identification of conserved and potentially regulatory small RNAs in heterocystous cyanobacteria, Front. Microbiol. 7 (2016) 48.

[52] B. Voss, J. Georg, V. Schön, S. Ude, W.R. Hess, Biocomputational prediction of non-coding RNAs in model cyanobacteria, BMC Genomics 10 (2009) 123.

[53] M. Kopf, W.R. Hess, Regulatory RNAs in photosynthetic cyanobacteria, FEMS Microbiol. Rev. 39 (2015) 301-315.

[54] A.S. Richter, C. Schleberger, R. Backofen, C. Steglich, Seed-based IntaRNA prediction combined with GFP-reporter system identifies mRNA targets of the small RNA Yfr1, Bioinformatics 26 (2010) 1-5.

[55] T. Nakamura, K. Naito, N. Yokota, C. Sugita, M. Sugita, A cyanobacterial noncoding RNA, Yfr1, is required for growth under multiple stress conditions, Plant Cell Physiol 48 (2007) 1309-1318.

[56] C. Steglich, M.E. Futschik, D. Lindell, B. Voss, S.W. Chisholm, W.R. Hess, The challenge of regulation in a minimal photoautotroph: non-coding RNAs in Prochlorococcus, PLoS Genet. 4 (2008) e1000173.

[57] J. Georg, W.R. Hess, Regulatory RNAs in cyanobacteria: developmental decisions, stress responses and a plethora of chromosomally encoded cis-antisense RNAs, Biol. Chem. 392 (2011) 291-297.

[58] M. Brenes-Álvarez, A. Vioque, A.M. Muro-Pastor, The integrity of the cell wall and its remodeling during heterocyst differentiation are regulated by phylogenetically conserved small RNA Yfr1 in Nostoc sp. strain PCC 7120, MBio (2020), https://doi. org/10.1128/mBio.02599-19 11:e02599-19, (in press).

[59] S.J. Lambrecht, Y. Kanesaki, J. Fuss, B. Huettel, R. Reinhardt, C. Steglich, Interplay and targetome of the two conserved cyanobacterial sRNAs Yfr1 and Yfr2 in Prochlorococcus MED4, Sci. Rep. 9 (2019) 14331.

[60] S.J. Lambrecht, J.M.L. Wahlig, C. Steglich, The GntR family transcriptional regulator PMM1637 regulates the highly conserved cyanobacterial sRNA Yfr2 in marine picocyanobacteria, DNA Res. 25 (2018) 489-497.

[61] L. Salmena, L. Poliseno, Y. Tay, L. Kats, P.P. Pandolfi, A ceRNA hypothesis: the Rosetta stone of a hidden RNA language? Cell 146 (2011) 353-358.

[62] Y. Tay, J. Rinn, P.P. Pandolfi, The multilayered complexity of ceRNA crosstalk and competition, Nature 505 (2014) 344-352.

[63] L. Bossi, N. Figueroa-Bossi, Competing endogenous RNAs: a target-centric view of small RNA regulation in bacteria, Nat. Rev. Microbiol. 14 (2016) 775-784.

[64] G. Gierga, B. Voss, W.R. Hess, Non-coding RNAs in marine Synechococcus and their regulation under environmentally relevant stress conditions, ISME J 6 (2012) $1544-1557$.

[65] M.A. Hernández-Prieto, V. Schön, J. Georg, L. Barreira, J. Varela, W.R. Hess, M.E. Futschik, Iron deprivation in Synechocystis: inference of pathways, noncoding RNAs, and regulatory elements from comprehensive expression profiling, G3 (Bethesda) 2 (2012) 1475-1495.

[66] J.A. Hernández, A.M. Muro-Pastor, E. Flores, M.T. Bes, M.L. Peleato, M.F. Fillat, Identification of a furA cis antisense RNA in the cyanobacterium Anabaena sp. PCC 7120, J. Mol. Biol. 355 (2006) 325-334.

[67] J.A. Hernández, I. Alonso, S. Pellicer, M. Luisa Peleato, R. Cases, R.J. Strasser F. Barja, M.F. Fillat, Mutants of Anabaena sp. PCC 7120 lacking alr1690 and alphafurA antisense RNA show a pleiotropic phenotype and altered photosynthetic machinery, J. Plant Physiol. 167 (2010) 430-437.

[68] E. Sevilla, B. Martín-Luna, A. González, J.A. Gonzalo-Asensio, M.L. Peleato, M.F. Fillat, Identification of three novel antisense RNAs in the fur locus from unicellular cyanobacteria, Microbiology 157 (2011) 3398-3404.

[69] B. Martín-Luna, E. Sevilla, A. González, M.T. Bes, M.F. Fillat, M.L. Peleato, Expression of fur and its antisense $\alpha$ - fur from Microcystis aeruginosa PCC7806 as response to light and oxidative stress, J. Plant Physiol. 168 (2011) 2244-2250.

[70] U. Dühring, I.M. Axmann, W.R. Hess, A. Wilde, An internal antisense RNA regulates expression of the photosynthesis gene isiA, Proc. Natl. Acad. Sci. U. S. A. 103 (2006) 7054-7058.

[71] S. Legewie, D. Dienst, A. Wilde, H. Herzel, I.M. Axmann, Small RNAs establish delays and temporal thresholds in gene expression, Biophys. J. 95 (2008) $3232-3238$

[72] J. Georg, W.R. Hess, cis-antisense RNA, another level of gene regulation in bac teria, Microbiol. Mol. Biol. Rev. 75 (2011) 286-300.

[73] A.D. Kappell, L.G. van Waasbergen, The response regulator RpaB binds the high light regulatory 1 sequence upstream of the high-light-inducible $h l i B$ gene from the cyanobacterium Synechocystis PCC 6803, Arch. Microbiol. 187 (2007) 337-342.

[74] M.L. López-Redondo, F. Moronta, P. Salinas, J. Espinosa, R. Cantos, R. Dixon, A. Marina, A. Contreras, Environmental control of phosphorylation pathways in a branched two-component system: NblS, a branched two-component system, Mol. 
Microbiol. 78 (2010) 475-489.

[75] M. Riediger, Y. Hihara, W.R. Hess, From cyanobacteria and algae to land plants: the RpaB/Ycf27 regulatory network in transition, Perspectives in Phycology 5 (2018) 13-25.

[76] M. Riediger, T. Kadowaki, R. Nagayama, J. Georg, Y. Hihara, W.R. Hess, Biocomputational analyses and experimental validation identify the regulon controlled by the redox-responsive transcription factor RpaB, IScience 15 (2019) 316-331.

[77] J. Georg, G. Kostova, L. Vuorijoki, V. Schön, T. Kadowaki, T. Huokko, D. Baumgartner, M. Müller, S. Klähn, Y. Allahverdiyeva, Y. Hihara, M.E. Futschik, E.-M. Aro, W.R. Hess, Acclimation of oxygenic photosynthesis to iron starvation is controlled by the sRNA IsaR1, Curr. Biol. 27 (2017) 1425-1436.e7.

[78] E. Massé, C.K. Vanderpool, S. Gottesman, Effect of RyhB small RNA on global iron use in Escherichia coli, J. Bacteriol. 187 (2005) 6962-6971.

[79] V. Krynická, M. Tichý, J. Krafl, J. Yu, R. Kaňa, M. Boehm, P.J. Nixon, J. Komenda, Two essential FtsH proteases control the level of the Fur repressor during iron deficiency in the cyanobacterium Synechocystis sp. PCC 6803, Mol. Microbiol. 94 (2014) 609-624.

[80] V. Krynická, J. Georg, P.J. Jackson, M.J. Dickman, C.N. Hunter, M.E. Futschik, W.R. Hess, J. Komenda, Depletion of the FtsH1/3 proteolytic complex suppresses the nutrient stress response in the cyanobacterium Synechocystis PCC 6803, Plant Cell 31 (2019) 2912-2928, https://doi.org/10.1105/tpc.19.00411 (press).

[81] H. Rübsam, F. Kirsch, V. Reimann, A. Erban, J. Kopka, M. Hagemann, W. R. Hess, S. Klähn, The iron-stress activated RNA 1 (IsaR1) coordinates osmotic acclimation and iron starvation responses in the cyanobacterium Synechocystis sp. PCC 6803, Environ. Microbiol. 20 (2018) 2757-2768.

[82] I. Sakurai, D. Stazic, M. Eisenhut, E. Vuorio, C. Steglich, W.R. Hess, E.-M. Aro, Positive regulation of $p s b A$ gene expression by cis-encoded antisense RNAs in Synechocystis sp. PCC 6803, Plant Physiol. 160 (2012) 1000-1010.

[83] J. Hu, T. Li, W. Xu, J. Zhan, H. Chen, C. He, Q. Wang, Small antisense RNA RblR positively regulates RuBisCo in Synechocystis sp. PCC 6803, Front. Microbiol. 8 (2017).

[84] M. Eisenhut, J. Georg, S. Klähn, I. Sakurai, H. Mustila, P. Zhang, W.R. Hess, E.M. Aro, The antisense RNA As1 flv4 in the Cyanobacterium Synechocystis sp. PCC 6803 prevents premature expression of the flv4-2 operon upon shift in inorganic carbon supply, J. Biol. Chem. 287 (2012) 33153-33162.

[85] J. Georg, D. Dienst, N. Schürgers, T. Wallner, D. Kopp, D. Stazic, E. Kuchmina, S. Klähn, H. Lokstein, W.R. Hess, A. Wilde, The small regulatory RNA SyR1/PsrR1 controls photosynthetic functions in cyanobacteria, Plant Cell 26 (2014) 3661-3679.

[86] T. Kadowaki, R. Nagayama, J. Georg, Y. Nishiyama, A. Wilde, W.R. Hess, Y. Hihara, A feed-forward loop consisting of the response regulator RpaB and the small RNA PsrR1 controls light acclimation of photosystem I gene expression in the cyanobacterium Synechocystis sp. PCC 6803, Plant Cell Physiol 57 (2016) $813-823$.

[87] J. Giner-Lamia, R. Robles-Rengel, M.A. Hernández-Prieto, M.I. Muro-Pastor, F.J. Florencio, M.E. Futschik, Identification of the direct regulon of NtcA during early acclimation to nitrogen starvation in the cyanobacterium Synechocystis sp. PCC 6803, Nucleic Acids Res. 45 (2017) 11800-11820.

[88] S. Klähn, I. Orf, D. Schwarz, J.K.F. Matthiessen, J. Kopka, W.R. Hess, M. Hagemann, Integrated transcriptomic and metabolomic characterization of the low-carbon response using an $n d h R$ mutant of Synechocystis sp. PCC 6803, Plant Physiol. 169 (2015) 1540-1556.

[89] I. Orf, S. Klähn, D. Schwarz, M. Frank, W.R. Hess, M. Hagemann, J. Kopka, Integrated analysis of engineered carbon limitation in a quadruple $\mathrm{CO}_{2} / \mathrm{HCO}^{3-}$ uptake mutant of Synechocystis sp. PCC 6803, Plant Physiol. 169 (2015) $1787-1806$.

[90] I. Álvarez-Escribano, A. Vioque, A.M. Muro-Pastor, NsrR1, a nitrogen stress-repressed sRNA, contributes to the regulation of nblA in Nostoc sp. PCC 7120, Front Microbiol. 9 (2018) 2267.

[91] S. Klähn, C. Schaal, J. Georg, D. Baumgartner, G. Knippen, M. Hagemann, A.M. Muro-Pastor, W.R. Hess, The sRNA NsiR4 is involved in nitrogen assimilation control in cyanobacteria by targeting glutamine synthetase inactivating factor IF7, Proc. Natl. Acad. Sci. U. S. A. 112 (2015) E6243-E6252.

[92] M. García-Domínguez, J.C. Reyes, F.J. Florencio, Glutamine synthetase inactivation by protein-protein interaction, Proc. Natl. Acad. Sci. U. S. A. 96 (1999) 7161-7166.

[93] T.D. Ames, R.R. Breaker, Bacterial aptamers that selectively bind glutamine, RNA Biol. 8 (2011) 82-89.

[94] L. Huang, J. Wang, A.M. Watkins, R. Das, D.M. Lilley, Structure and ligand binding of the glutamine-II riboswitch, Nucleic Acids Res. 47 (2019) 7666-7675.

[95] E. Flores, S. Picossi, A. Valladares, A. Herrero, Transcriptional regulation of development in heterocyst-forming cyanobacteria, Biochim Biophys Acta Gene Regul Mech 1862 (2019) 673-684.

[96] A.M. Muro-Pastor, A. Valladares, E. Flores, A. Herrero, Mutual dependence of the expression of the cell differentiation regulatory protein HetR and the global nitrogen regulator NtcA during heterocyst development, Mol. Microbiol. 44 (2002) 1377-1385.

[97] M. Brenes-Álvarez, J. Mitschke, E. Olmedo-Verd, J. Georg, W.R. Hess, A. Vioque, A.M. Muro-Pastor, Elements of the heterocyst-specific transcriptome unravelled by co-expression analysis in Nostoc sp. PCC 7120, Environ. Microbiol. 21 (2019) $2544-2558$.

[98] A.M. Muro-Pastor, The heterocyst-specific NsiR1 small RNA is an early marker of cell differentiation in cyanobacterial filaments, mBio 5 (2014) e01079-01014.

[99] P. Mandin, M. Guillier, Expanding control in bacteria: interplay between smal RNAs and transcriptional regulators to control gene expression, Curr. Opin. Microbiol. 16 (2013) 125-132.

[100] M. Nitzan, R. Rehani, H. Margalit, Integration of bacterial small RNAs in regulatory networks, Annu. Rev. Biophys. 46 (2017) 131-148.

[101] A.J. de Porcellinis, S. Klähn, L. Rosgaard, R. Kirsch, K. Gutekunst, J. Georg, W.R. Hess, Y. Sakuragi, The non-coding RNA Ncr0700/PmgR1 is required for photomixotrophic growth and the regulation of glycogen accumulation in the cyanobacterium Synechocystis sp. PCC 6803, Plant Cell Physiol 57 (2016) 2091-2103.

[102] K. Voigt, C.M. Sharma, J. Mitschke, S. Joke Lambrecht, B. Voß, W.R. Hess, C. Steglich, Comparative transcriptomics of two environmentally relevant cyanobacteria reveals unexpected transcriptome diversity, ISME J 8 (2014) 2056-2068.

[103] R. Lorenz, S.H. Bernhart, C. Höner zu Siederdissen, H. Tafer, C. Flamm, P.F. Stadler, I.L. Hofacker, ViennaRNA package 2.0, Algorithms for Molecular Biology 6 (2011).

[104] K. Darty, A. Denise, Y. Ponty, VARNA: interactive drawing and editing of the RNA secondary structure, Bioinformatics 25 (2009) 1974-1975. 